\title{
Verification of Volume Similarity Between Unilateral Mammary Gland and Autologous Omentum in Adult Women by Measuring Cylinder Method
}

\author{
Guangtai Shen' \\ Xiaoqiang $\mathrm{Yu}^{\prime}$ \\ Tianyang Sun' \\ Tian Hou' \\ Heping $\mathrm{He}^{\prime}$ \\ Tubuxin Bai ${ }^{1}$ \\ Zhipeng Zhang' \\ Xiaoli Ding' \\ Qingping $\mathrm{Li}^{2}$ \\ Ying Liang ${ }^{3}$ \\ Yuhua $\mathrm{Ma}^{4}$ \\ 'Department of Breast and Thyroid \\ Surgery, Inner Mongolia Xing'an League \\ People's Hospital, Ulanhot, Inner \\ Mongolia, 137400, People's Republic of \\ China; ${ }^{2}$ Department of Operating Room, \\ Inner Mongolia Xing'an League People's \\ Hospital, Ulanhot, Inner Mongolia, \\ 137400, People's Republic of China; \\ ${ }^{3}$ Department of Pathology, Inner \\ Mongolia Xing'an League People's \\ Hospital, Ulanhot, Inner Mongolia, \\ 137400, People's Republic of China; \\ ${ }^{4}$ Department of Anesthesiology, Inner \\ Mongolia Xing'an League People's \\ Hospital, Ulanhot, Inner Mongolia, \\ 137400, People's Republic of China
}

Correspondence: Xiaoqiang Yu Department of Breast and Thyroid Surgery, Inner Mongolia Xing'an League People's Hospital, Ulanhot, Inner Mongolia, I37400,

People's Republic of China

Tel/Fax +86 0482-84I376I

Email yuxq66_dr@I63.com
Objective: To verify the volume similarity between unilateral mammary gland and autologous omentum in adult females.

Methods: A total of 63 patients diagnosed with stage 0-II breast cancer and partial nonlactating multi-fistula mastitis in the breast surgery department of Inner Mongolia Xing'an League People's Hospital from 2007 to 2020 were enrolled in the study, including 52 cases of stage $0-$ II breast cancer and 11 cases of non-lactating multi-fistula mastitis. The volume of the resected mammary gland and the omentum were measured by a "soft tissue measuring cylinder" and recorded. The appearance of the reconstructed breast was compared with that of the healthy side. The correlation between unilateral mammary gland volume and autologous omentum volume was analyzed by linear regression.

Results: Valid data were obtained for 60 cases. Affected breast size, curve, texture, nipple, and inframammary fold after omentum breast reconstruction were similar and symmetrical to those of the unaffected side. Postoperative complications occurred in most patients; the majority of these $(76.67 \%)$ involved numbness of the nipple, and other complications were few. Patient satisfaction with postoperative appearance, feel, and movement of the breast, as well as total treatment costs, was over $75.0 \%$. Linear regression analysis indicates a linear relationship between subcutaneous gland volume (x) and autologous omentum volume (y): $\mathrm{y}=0.9847 \mathrm{x}-1.2132, R^{2}=0.9742$.

Conclusion: Only when the dissociated pedicled omentum is completely obtained under laparoscopy can the whole subcutaneous residual cavity of the mammary gland be filled to the same volume. This study verifies that the volume of the unilateral mammary gland is similar to that of the autologous omentum in adult females.

Keywords: unilateral mammary gland, omentum, volume, similarity, laparoscope, breast reconstruction

\section{Introduction}

Current surgical methods of breast reconstruction in China and abroad include prosthetic breast reconstruction, autologous breast reconstruction, prosthesis combined with autologous breast reconstruction, and others. Autologous omentum breast reconstruction is an emerging minimally invasive surgical method. ${ }^{1}$ Omentum breast reconstruction is used most commonly in Phase I breast reconstruction after mammectomy (which is mainly concerned with the repair of defects 
in breast-conserving surgery), but is also used in complete mammectomy, filling of mammary gland residual cavity, and repair of defects after non-lactating mastitis resection. ${ }^{2}$ The omentum can be obtained by traditional laparotomy and laparoscopy. Compared with laparoscopic surgery, traditional laparotomy does not obtain sufficient omentum to reconstruct the breast well. The relationship between omentum and recipient site can either dissociated or pedicled. Although omentum breast reconstruction does not yet occupy the dominant position in breast reconstruction, it can exert its greatest advantages and reduce complications when the optimal indications are selected and delicate surgical techniques are applied. ${ }^{3}$ Meanwhile, most current studies evaluated the use of omentum greater for breast reconstruction from the surgical efficacy, but there were few reports on whether the volume of omentum greater was suitable for breast reconstruction. Therefore, this study aimed to compare the greater omentum volume measured during surgery with the volume of mammary gland to evaluate the surgical efficacy and provide guidance for clinical practice.

\section{Materials and Methods}

\section{Clinical Data}

A total of 63 patients diagnosed with stage 0-II breast cancer and partial non-lactating multi-fistula mastitis in our department from 2007 to 2020 were enrolled, including 52 cases of stage $0-$ II breast cancer (four cases with carcinoma in situ; the rest with invasive ductal carcinoma) and 11 cases of non-lactating multi-fistula mastitis. Three patients were lost to follow-up, so a total of 60 valid cases were obtained.

Inclusion criteria for patients were as follows: 1) prestage IIb breast cancer (multifocal carcinoma and carcinoma in situ) or chronic mastitis (multifocal mammary fistula); 2) no history of upper abdominal surgery; 3) no history of peritonitis; and 4) informed consent form signed and participation in this study volunteered.

Exclusion criteria were as follows: 1) history of midupper abdominal surgery or inflammatory lesions; 2) history of abdominal malignant tumors; 3) accompanying multiple organ lesions; 4) connective tissue disease; 5) advanced age or poor body function; and 6) severe obesity.

The patients were aged between 27 and 57. There were 62 unilateral cases and one bilateral case; all patients received a pathological diagnosis through puncture before surgery. For all patients, dissociated pedicled omentum was obtained under laparoscopy, and to reconstruct the breast after complete subcutaneous (unilateral) mammectomy, the residual cavity was filled to the same volume by the pedicled omentum.

\section{Surgical Methods}

In this section, we detail the methods of the consecutive surgical steps taken and the measures recommended to prevent complications.

\section{Subcutaneous Mammectomy and Tunnel Building}

The nipple and areola were first treated to ensure their blood supply. Using the "single forceps technique" (Figure 1A), the visual venous network below the areola was retained with a nipple-areola thickness of not less than $0.5 \mathrm{~cm}$. A tissue slice was cut under the nipple for rapid pathological examination (where there was no tumor infiltration, the nipple was preserved; otherwise, it was reconstructed with areola). ${ }^{4}$

The blood supply of the flap was protected, and the operation was gentle and precise. The key is to separate the coarse pellet fat from the gland, thus ensuring the thickness of the "coated capsule".

Three deltoid ligaments were protected: the subclavian deltoid ligament, parasternal deltoid ligament, and inframammary crease ligament. It should be noted that these three deltoid ligaments are of great importance to the appearance of the breast after reconstruction, acting as a three-walled frame (Figure 1B).

The volume of the subcutaneous gland was measured by a "soft tissue measuring cylinder" (a self-developed utility model patented instrument) (Figure 1C) and recorded. The breast body was installed into the laparoscopic plastic sleeve, and then loaded into the utility model patent cylinder measuring device to measure its volume, so as to avoid the spread of tumor.

A tunnel was built (Figure 1D). The superior margin of the rectus abdominis was found through the inframammary fold. The anterior sheath of the rectus abdominis was cut longitudinally $3 \mathrm{~cm}$ from the midline, and the rectus abdominis was separated obliquely to the midline. The tunnel was opened to a width of two to three transverse fingers. Care was taken at this stage not to open the peritoneum, to prevent the pneumoperitoneum from leaking. Oblique separation of the rectus abdominis is necessary to effectively prevent the occurrence of incisional hernia. ${ }^{5}$

\section{Dissociated Omentum Under Laparoscopy}

The course of the left, middle and right main vessels of the omentum was noted, as well as the presence of tumors and 

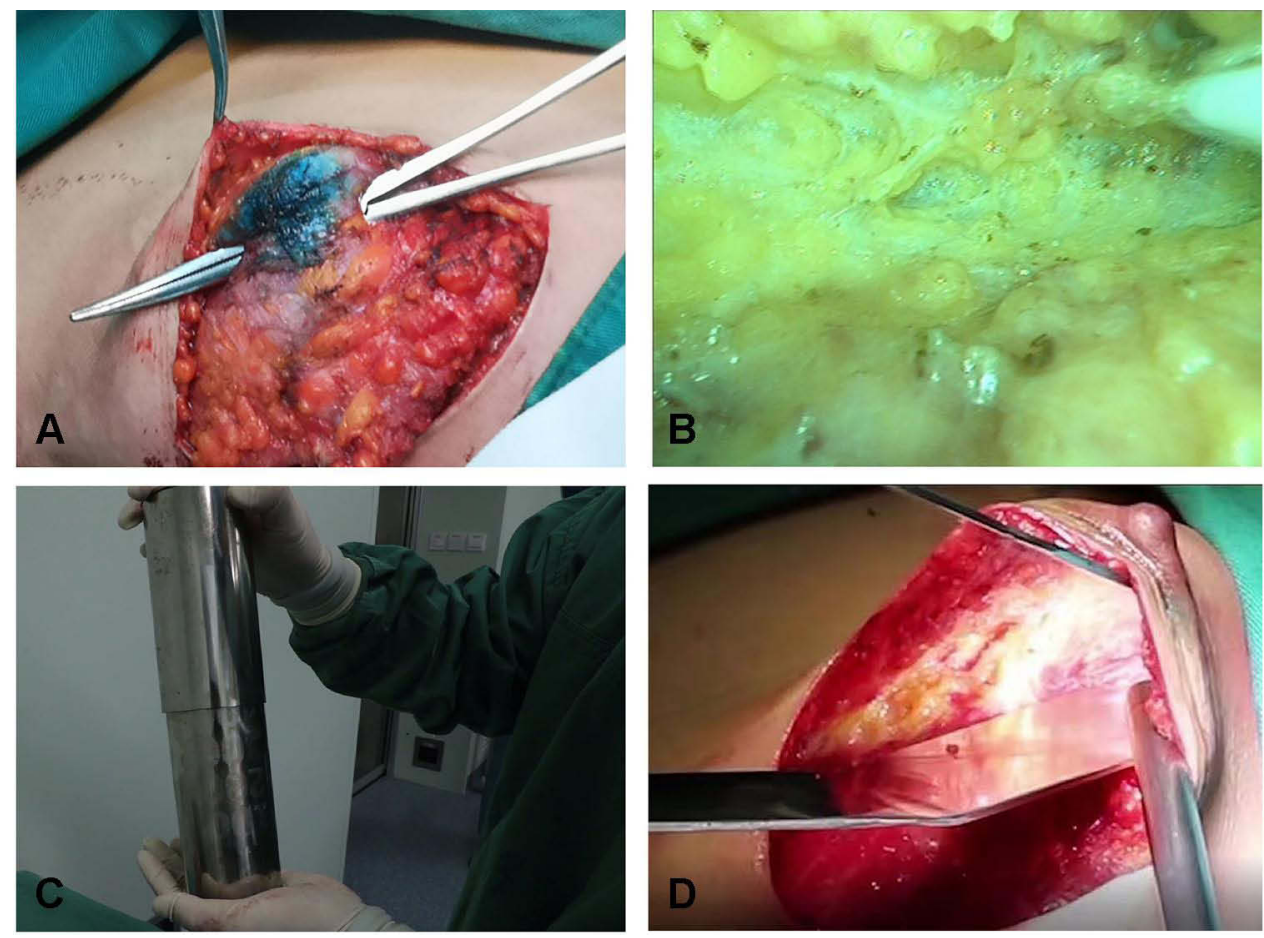

Figure I Subcutaneous mammectomy and tunnel building. (A) Visual venous network below the areola preserved by single forceps technique. (B) Three deltoid ligaments: subclavian deltoid ligament, parasternal deltoid ligament, and inframammary crease ligament. (C) Self-developed utility model patented instrument "soft tissue measuring cylinder" is used to measure subcutaneous gland volume. (D) Building the tunnel.

serious adhesion in the abdominal cavity. Then, the gastric antrum was exposed. The gastric branches of the gastric omental vessels were bundled and an ultrasonic scalpel was used to sever them from right to left with a starting point on the right side below the gastric antrum (Figure 2A), ${ }^{6}$ and an end point on the left side in front of the spleen. Care was taken to avoid burning the gastric wall or causing hemorrhage of the collateral branch of the gastric omental vessels. In order to prevent bleeding from the closed vessel stump of the gastric wall with gastric dilatation after eating, the lower gear of the ultrasonic scalpel was used.

At the middle part of the transverse colon, the gastrocolic ligament was lifted by the "curtain-pulling" method (Figure 2B). As there is no important blood vessel here, the left side could be severed from right to left (using the higher gear of the ultrasonic scalpel) and turned upward at the area in front of the hilus lienis. The adhesion here was often severe and hard to distinguish, so the severance was performed carefully. After severance, the initial part of the left blood vessel of the gastric omentum could be seen. Care was taken to protect the root of this vessel from accidental injury. ${ }^{2}$ The initial part of the left blood vessel of the gastric omentum was severed by transitional coagulation severance with the ultrasonic scalpel in low gear (Figure 2C), and the proximal end was clipped with a biological clip if necessary (Figure 2D). ${ }^{7}$

When the right half gastrocolic ligament was severed, care was taken to avoid injuring the root of the right vessel of the gastric omentum, ${ }^{8}$ by constantly observing the pulsation of this blood vessel. Then, the pedicled omentum was dissociated (Figure 2E). The lower left part of the omentum was clamped with non-invasive forceps to ensure smooth withdrawal from the tunnel.

\section{Withdrawing the Omentum}

Non-destructive forceps were inserted into the plastic sleeve of a laparoscope with a length of $50 \mathrm{~cm}$. The front edge of the sleeve mouth was reversely folded several times, and the margin of the sleeve mouth was clamped with the forceps; the forceps were then inserted through the outer opening of the tunnel and pushed toward the abdominal cavity from the outside of the peritoneum. At this time, hemispheric protrusion caused by external pressure was sometimes observed in the abdominal cavity: a peritoneum with hemispheric protrusion was longitudinally incised with the ultrasonic scalpel. The nondestructive forceps together with the laparoscopic sleeve were inserted into the abdominal cavity for about $30 \mathrm{~cm}$, 

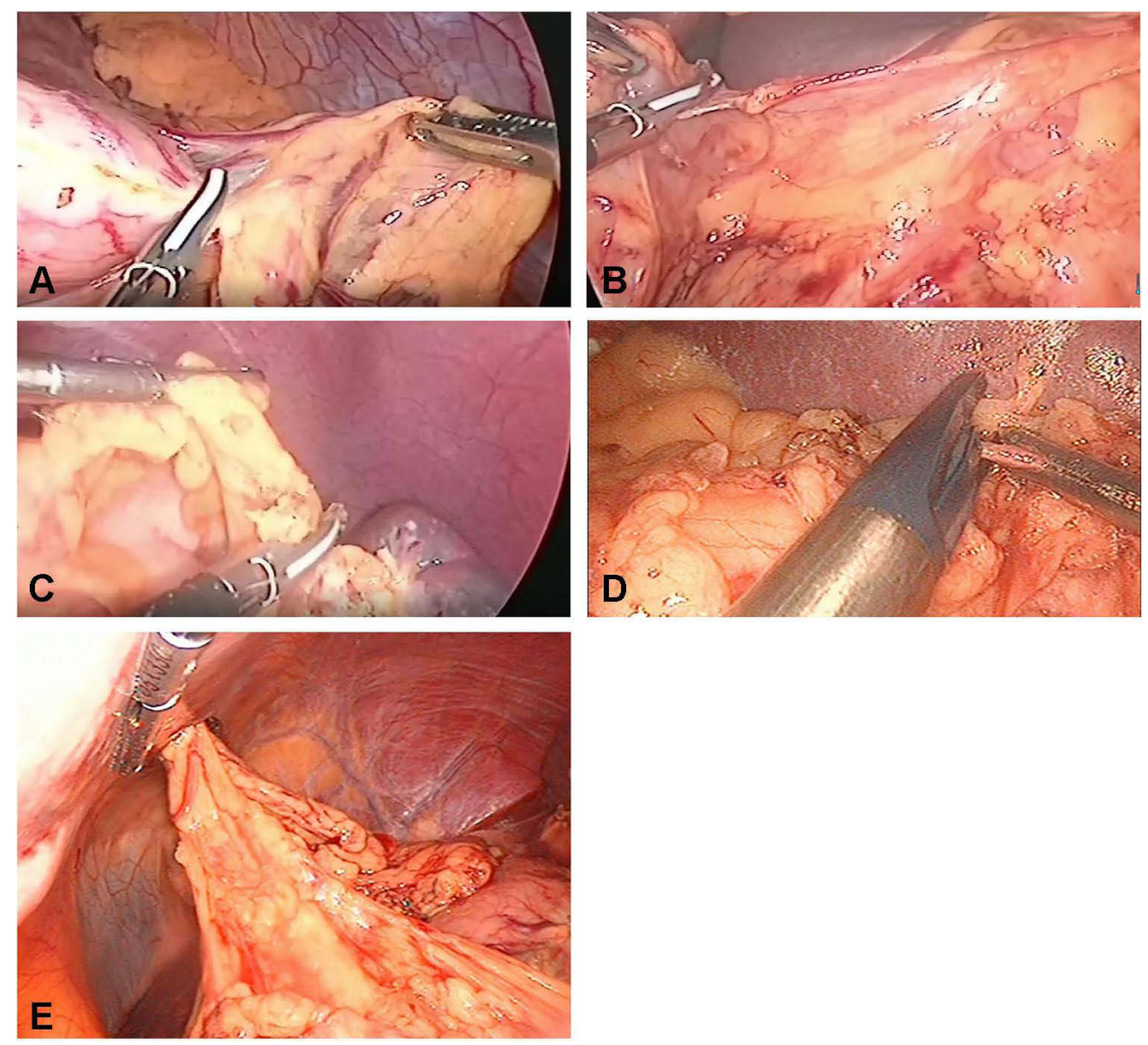

Figure 2 Dissociated omentum. (A) Gastric branch of severed gastric omental vessels. (B) The gastrocolic ligament in the middle of the transverse colon is lifted by the "curtain-pulling" method. (C) The initial part of the left blood vessel of the gastric omentum is severed by transitional coagulation severance with the ultrasonic scalpel in low gear. (D) The proximal end of the left blood vessel of the gastric omentum is clipped with a biological clip. (E) The pedicled omentum is dissociated.

and the non-destructive forceps were expanded; the forceps clamping the lower left part of the omentum were removed, and the lower left part of the omentum was transferred to the non-destructive forceps and clipped; the omentum was gently dragged into and through the laparoscopic sleeve (in order to reduce crush injury and contusion to the omentum) and pulled out of the abdominal cavity (Figure 3A). When slight resistance was encountered, the non-destructive forceps were released, and the laparoscopic sleeve and the part of the omentum outside the abdominal cavity were grasped and pulled out, and the pedicled part loosened without twisting. ${ }^{9}$ The abdominal cavity was checked for bleeding and burning.

To prevent the occurrence of tunnel hernia, the tunnel opening (anterior sheath of the rectus abdominis) and the inframammary fold opening were sutured to the point where two transverse fingers could be accommodated. ${ }^{2}$ The inframammary fold was reconstructed.

The vascular continuity of the omentum was observed against the light and was removed immediately in the case of any ischemia. The omentum was loaded into the laparoscopic sleeve and then into the soft tissue measuring cylinder for its volume to be measured and recorded (Figure 3B).

\section{Breast Reconstruction}

After good blood supply to the omentum was confirmed, the omentum was placed into the residual cavity of the breast in a coil. A drainage catheter was inserted, and the incision was sutured.

One day after surgery, the patient was asked to frequently sit up and undertake off-bed activities. The inframammary fold and lateral radian of the breast were manually adjusted to make the lateral radian as plump as possible, and smooth blood supply to the nipple was observed. ${ }^{10}$

The axillary drainage was positioned at the point where the midaxillary line met the nipple level. The drainage tube of reconstructed breast residual cavity was placed in the greater omentum and subcutaneous space, the position 

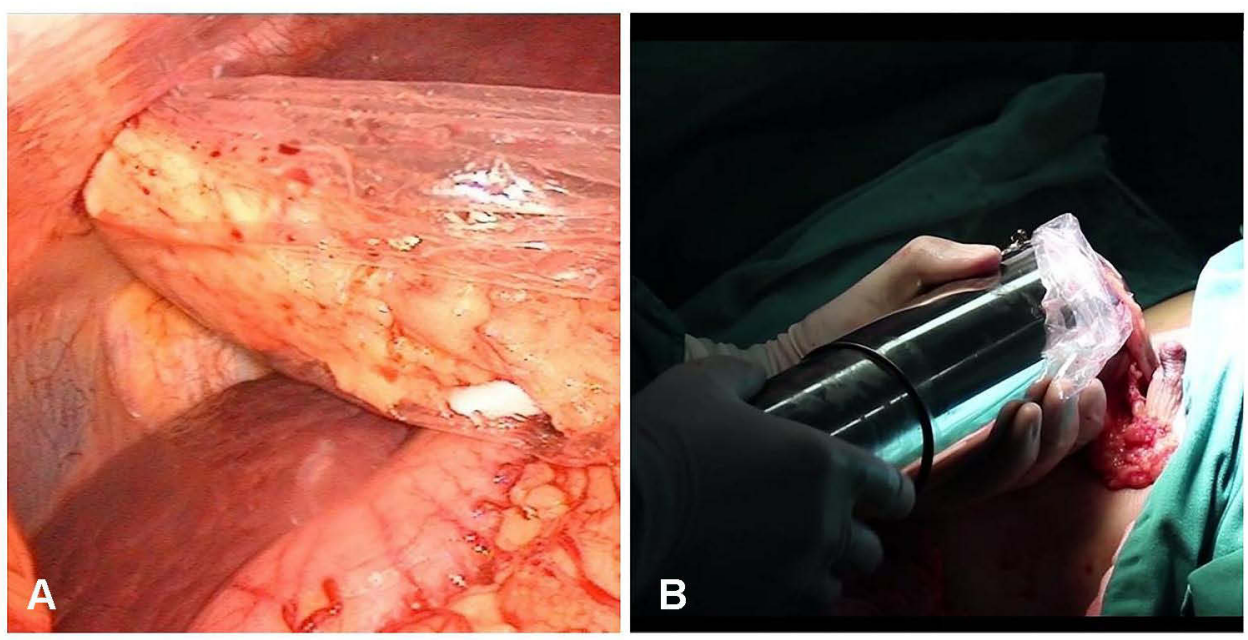

Figure 3 Withdrawing the omentum. (A) Part of the omentum is pulled out of the abdominal cavity through the laparoscopic sleeve. (B) The volume of the omentum is measured by the soft tissue measuring cylinder.

was the junction between the lowest level of the submammary fold and the midaxillary line. Meanwhile, normal saline injection containing 1:1000 concentration of epinephrine was injected into the drainage tunnel to prevent bleeding.

\section{Prevention of Complications}

The coarse pellet fat is separated from the gland, thus retaining a certain thickness of fat; in addition, the visual venous network below the areola should be preserved as much as possible to prevent ischemia of the nipple and areola. The pulsation of the blood vessel of the right main pedicle of the gastric omentum must be observed at all times as the right gastrocolic ligament is severed. If the blood vessel is damaged, the omentum should be taken out. ${ }^{11}$ Ultrasonic examination should be performed as early as possible to determine blood supply; early use of anticoagulants has a good effect on the formation of intravascular coagulation. If necrosis is confirmed, excision should be performed as early as possible. ${ }^{12}$ Vasodilators and anticoagulants can be used in the early stages, ${ }^{13}$ and manual massage can be performed to prevent Formation of hard nodules. Prevention of postoperative gastric distention and fullness involves confirming that the pedicle is loose and does not press against the gastric antrum. ${ }^{14}$

\section{Statistical Method}

Descriptive analysis and linear regression analysis were performed for the collected data in this study using SPSS20.0 software. The correlation analysis was completed by subordinating the volume of the subcutaneous gland (independent variable, $\mathrm{x}$ ) and that of the autologous omentum (dependent variable, y) into the linear regression equation.

\section{Results}

\section{Occurrence of Complications and Patient Satisfaction Survey}

The follow-up visit revealed that of the 63 patients who had undergone breast reconstruction, 62 cases were unilateral and one case was bilateral. Three patients were lost to follow-up, so a total of 60 valid cases were obtained. The results show that postoperative complications occurred in most patients; the majority of these $(76.67 \%)$ involved numbness of the nipple, and other complications were few (Table 1). Patient satisfaction with postoperative appearance, feel, and movement of the breast, as well as expense, was over $75.0 \%$ (Table 2 ).

\section{Relationship Between Volumes of Subcutaneous Gland and Autologous Omentum}

We assessed symmetry by the appearance of the breast and nipple. The criteria of breast symmetry: bilateral breast size, curve, upper half, lower half were symmetric. The criteria of nipple symmetry: both nipples were at the same level and the distance between the bilateral papilla and the median line was equal. The results show that affected breast size, curve, texture, nipple, and lower plica level after omentum breast reconstruction were similar and symmetrical to those of the unaffected side (Figure 4). The linear regression analysis reveals a linear relationship between the volume of the subcutaneous gland (x) and 
Table I Complications After Breast Reconstruction $(n=60)$

\begin{tabular}{|l|c|c|}
\hline Classification & Number & Proportion (\%) \\
\hline Local recurrence & 2 & 3.33 \\
Tunnel hernia & 1 & 1.67 \\
Postoperative distention and fullness & 3 & 5.0 \\
Complete omentum necrosis & 2 & 3.33 \\
Mastoptosis & 3 & 5.0 \\
Hard nodules & 3 & 5.0 \\
Necrosis of skin margin & 1 & 1.67 \\
Necrosis of nipple and areola & 2 & 3.33 \\
Upper shift of the reconstructed nipple & 4 & 6.67 \\
Bulging of the inframammary fold & 2 & 3.3 \\
Numbness of the nipple & 46 & $76.67 \%$ \\
\hline
\end{tabular}

Table 2 Postoperative Satisfaction Survey $(n=60)$

\begin{tabular}{|l|c|c|}
\hline Satisfaction Index & Number & Proportion (\%) \\
\hline Appearance & 58 & 96.67 \\
Hand feeling and movement & 48 & 80.0 \\
Expense & 45 & 75.0 \\
\hline
\end{tabular}

that of the autologous omentum $(\mathrm{y}): \mathrm{y}=0.9847 \mathrm{x}-1.2132$, $R^{2}=0.9742$ (Figure 5).

\section{Discussion}

For adult women, a pedicled omentum with similar volume to that of the unilateral mammary gland can be obtained only when the omentum is fully dissociated under laparoscopy. ${ }^{15}$

The success of subcutaneous mammectomy and omentum breast reconstruction is determined by the following five factors. First, the three deltoid ligaments detailed in Subcutaneous Mammectomy and Tunnel Building are preserved in mammectomy. Second, when the omentum is dissociated under the laparoscope, damage to the dissociated vessels is avoided. The key to success is to dissociate the omentum with closed loop vessels. Third, the omentum is not subject to crush injury by the tunnel as it is withdrawn, and the volume of the resected mammary gland (as measured with the soft tissue measuring cylinder) is similar to that of the omentum. Fourth, complications such as intestinal burns, intestinal canal ischemia, splenic rupture, and ischemia of breast skin, nipple, and areola are avoided. The patient recovers quickly as early as possible, and the reconstructed breast has no swelling or pain. Good symmetry, softness to the touch and a high degree of patient satisfaction are the ideal standards. ${ }^{16}$

It is worth discussing the justification of the use of the omentum in breast reconstruction. The omentum is a part of the peritoneum that has functions in fighting disease, including infection prevention, absorption, and wrapping. In a stable physiological state, the omentum is relatively static and "waiting", and the probability that a person (in the general population) suffering from abdominal infectious diseases needs the omentum to move and wrap for infection prevention is very low. The incidence rate of breast cancer in the general population is about 40 in 100,000, of which operable breast cancer accounts for $93 \%$ (about 37.2 ). Less than $10 \%$ of the patients in the operable breast cancer population are suitable for omentum breast reconstruction. As the probability that any of these $10 \%$ will require their omentum for infection prevention is extremely low (as stated above), the omentum can be reasonably transferred and used as an organ in a relatively static and dormant state. This study finds that there is a close linear relationship between the volume of the unilateral mammary gland and the volume of the autologous omentum in adult females. Hence, in mammectomy, the residual cavity of the mammary gland can be appropriately filled by the autologous omentum, not only avoiding the rejection reaction caused by the application of exogenous materials but also ensuring symmetrical appearance. The use of the autologous omentum is therefore of great significance for breast cancer surgery.

\section{Limitations}

This study has some limitations and defects; it also points to potential directions for further research:

- The variation of gastric omental vessels is not considered. Routine abdominal CT angiography before operation should be studied.

- Future study should discuss supporting the survival of the omentum by anastomosis in the abdominal cavity with thoracic dorsal vessels and intercostal vessels when two major blood vessels are damaged.

- The influence of the location of drainage catheters on the drainage effect should be further investigated.

- Racial differences may affect the size of the greater omentum mammary gland, which needs to be further explored in multi-center studies.

- The need for pressure bandaging after operation should be studied further.

\section{Conclusion}

In this study, a close linear relationship was found between the volume of unilateral mammary gland and the volume of autologous omentum in adult women. It is suggested that the residual cavity of the breast can be properly filled 

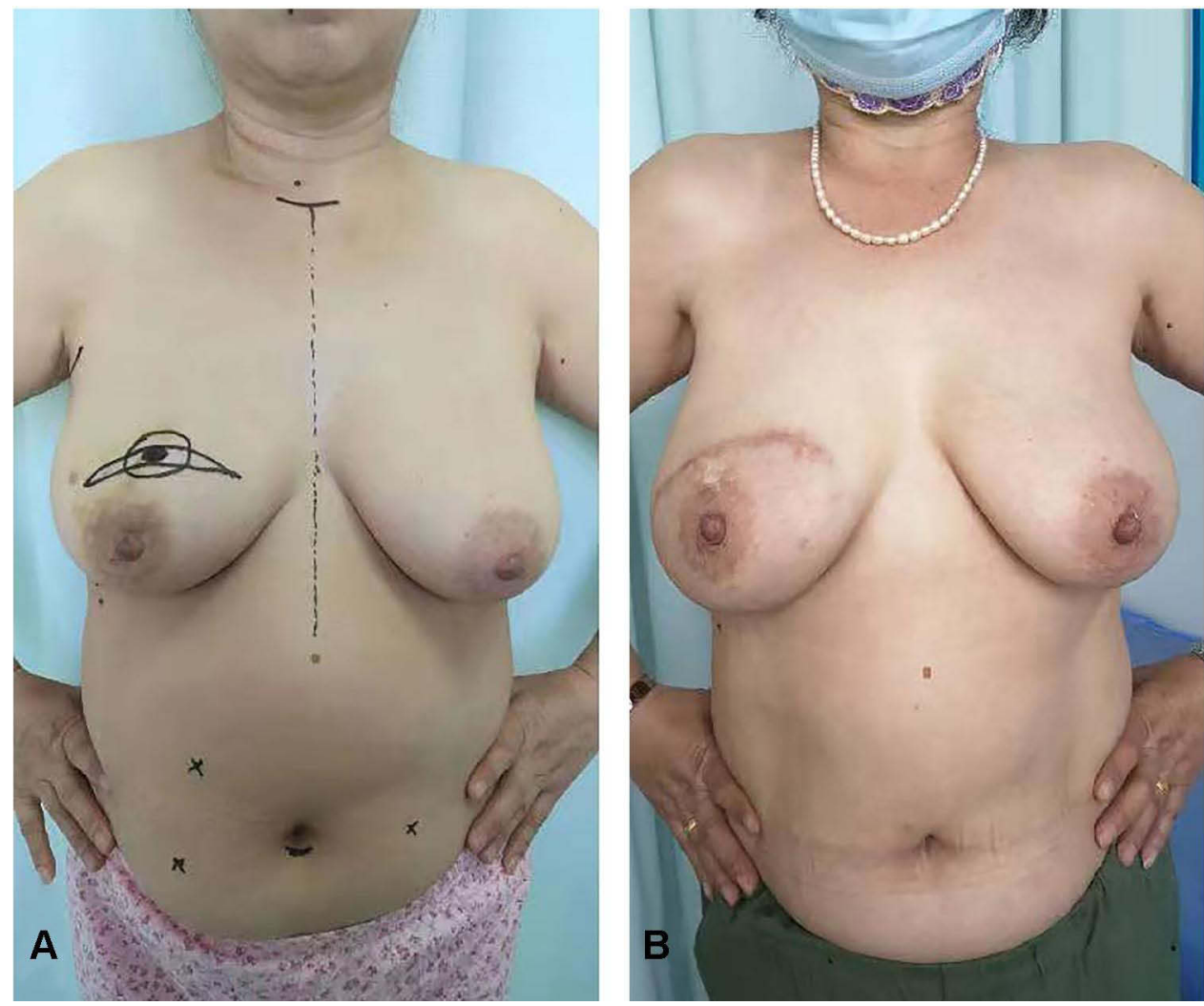

Figure 4 Comparison diagrams: before and after omentum breast reconstruction. (A) Before operation. (B) After operation.

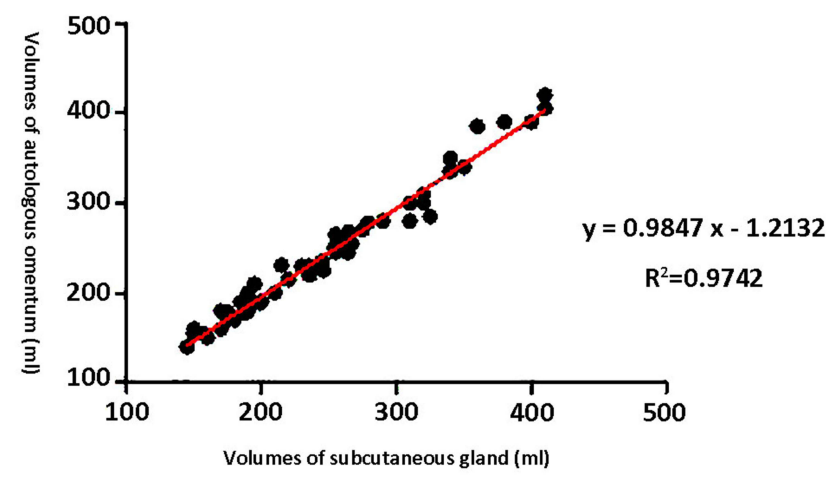

Figure 5 Linear relationship between the volumes of the subcutaneous gland and the autologous omentum.

by autologous omentum during mastectomy, which is of great significance for the application of autologous omentum in breast cancer surgery.

\section{Ethics Approval and Consent to Participate}

The study was conducted in accordance with the Declaration of Helsinki (as was revised in 2013). The study was approved by Ethics Committee of Inner
Mongolia Xing'an League People's Hospital. All patients were adequately informed and gave informed consent.

\section{Acknowledgments}

We are particularly grateful to all the people who have given us help on our article.

\section{Funding}

There is no funding to report.

\section{Disclosure}

The authors declare that they have no competing interests.

\section{References}

1. Zaha H, Onomura M, Nomura H, Umekawa K, Oki M, Asato H. Free omental flap for partial breast reconstruction after breast-conserving surgery. Plast Reconstr Surg. 2012;129(3):583-587. doi:10.1097/ PRS.0b013e3182402cc6

2. Song XY, Guan DD. Pedicle omentum flap harvested by laparoscopy in breast oncoplastic surgery China. Oncology. 2017;27(08):608-612. [Article in Chinese].

3. Wan N, Liu D, Lu L, et al. Application of pedicled omentum flap in breast reconstruction of breast cancer patients. Zhongguo Xiu Fu Chong Jian Wai Ke Za Zhi. 2019;33(8):1006-1010. doi:10.7507/ 1002-1892.201901007 
4. Guan D, Lin H, Lv Z, Xin Y, Meng K, Song X. The oncoplastic breast surgery with pedicled omental flap harvested by laparoscopy: initial experiences from China. World J Surg Oncol. 2015;13:95. doi:10.1186/s12957-015-0514-9

5. Zhang P, He L, Shi F, Deng J, Fang C, Luo Y. Three-dimensional visualization technique in endoscopic breast-conserving surgery and pedicled omentum for immediate breast reconstruction. Surg Oncol. 2019;28:103-108. doi:10.1016/j.suronc.2018.11.016

6. Li M, Liu XD, Hu JF, et al. Effectiveness of laparoscopic free tipped large omental breast filling for early stage breast cancer. Peoples Military Surg. 2017;60:355-357+360. [Article in Chinese].

7. Wang ZH, Zhang YL, Qu X. Breast reconstruction technique using large omental tissue. Chin J Pract Surg. 2019;39(11):1235-1237. [Article in Chinese].

8. Chen XC, Yan WT, Wu XJ, et al. Da Vinci robot-assisted filling witli pedicled omental flap for breast reconstruction. J Region Anatomy Operat Surg. 2017;26(11):823-826. [Article in Chinese].

9. Chen L, Xu SM. Minimally invasive breast reconstruction: literature review and new tendency. Chin J Breast Dis. 2016;10(3):129-132. [Article in Chinese].

10. Li N, Zheng $\mathrm{Z}$, Li J, et al. Immediate breast reconstruction with omental flap for luminal breast cancer patients: ten clinical case reports. Medicine. 2017;96(33):e7797. doi:10.1097/MD.000000 0000007797
11. Chen T, Jia LJ, Zhai BP, et al. Surgical methods of endoscopic breast reconstruction. Chin J Breast Dis. 2019;13(2):124-126. [Article in Chinese].

12. Ni C, Zhu Z, Xin Y, et al. Oncoplastic breast reconstruction with omental flap: a retrospective study and systematic review. $J$ Cancer. 2018;9(10):1782-1790. doi:10.7150/jca.25556

13. Shi XQ, Zheng C, Hu DH, et al. Standardized approach for immediate breast reconstruction with free omentum transplantation after breast cancer surgery. Med J Natl Defend Forces Northwest China. 2019;40(12):727-731.

14. Wang Z, Xin P, Zhang Z, et al. Application of laparoscopically harvested pedicled omental flap for breast reconstruction after breast cancer surgery. Chin J Breast Dis. 2019;13(02):65-68. [Article in Chinese].

15. Shen G, Yu X. Application value of laparoscopy in radical mastectomy and omental breast reconstruction. Oncol Lett. 2019;18 (1):645-650. doi:10.3892/ol.2019.10339

16. Li NL, Zheng $\mathrm{C}$, Li JP, et al. Immediate breast reconstruction with free omentum: 7 cases. Chin J Breast Dis. 2016;10(4):210-214. [Article in Chinese].
International Journal of General Medicine

\section{Publish your work in this journal}

The International Journal of General Medicine is an international, peer-reviewed open-access journal that focuses on general and internal medicine, pathogenesis, epidemiology, diagnosis, monitoring and treatment protocols. The journal is characterized by the rapid reporting of reviews, original research and clinical studies

\section{Dovepress}

across all disease areas. The manuscript management system is completely online and includes a very quick and fair peer-review system, which is all easy to use. Visit http://www.dovepress.com/ testimonials.php to read real quotes from published authors. 